\title{
Experimental investigation of nitrile formation from VUV photochemistry of interstellar ices analogs: acetonitrile and amino acetonitrile
}

\author{
G. Danger ${ }^{1}$, J.-B. Bossa ${ }^{1}$, P. de Marcellus ${ }^{2}$, F. Borget ${ }^{1}$, F. Duvernay $^{1}$, P. Theulé ${ }^{\text {, }}$ \\ T. Chiavassa ${ }^{1}$, and L. d'Hendecourt ${ }^{2,3}$ \\ ${ }^{1}$ Université de Provence, Laboratoire de Physique des Interactions Ioniques et Moléculaires Centre de St-Jérôme, \\ Avenue Escadrille Normandie-Niemen, 13397 Marseille, France \\ e-mail: gregoire.danger@univ-provence.fr \\ 2 Univ. Paris-Sud, Astrochimie et Origines, Institut dAstrophysique Spatiale, UMR 8617, Orsay, 91405, France \\ 3 CNRS, Orsay, 91405, France
}

Received 10 September 2010 / Accepted 4 October 2010

\section{ABSTRACT}

\begin{abstract}
Context. The study of the chemical reactivity in interstellar ices in astrophysical environments is an important tool for understanding the origin of the organic matter in molecular clouds, in protoplanetary disks, and possibly, as a final destination, in our solar system. The laboratory simulations of the reactivity in ice analogs provide important information for understanding the reactivity in these environments. Here, we used these experimental simulations to trace some formation pathways of two nitriles, acetonitrile and amino acetonitrile, which are two potential precursors of amino acids in astrophysical environments.

Aims. The purpose of this work is to present the first experimental approach for the formation of acetonitrile and amino acetonitrile in interstellar-like conditions.

Methods. We use Fourier Transform InfraRed (FTIR) spectroscopy and mass spectrometry to study the formation at $20 \mathrm{~K}$ of acetonitrile $\mathrm{CH}_{3} \mathrm{CN}$ from VUV irradiation of ethylamine and of amino acetonitrile $\mathrm{NH}_{2} \mathrm{CH}_{2} \mathrm{CN}$ from VUV irradiation of ammonia: acetonitrile mixture. Isotopic substitutions are used to confirm identifications.

Results. We demonstrate that acetonitrile can be formed at $20 \mathrm{~K}$ from the VUV irradiation of ethylamine with a yield of $4 \%$. Furthermore, in presence of ammonia, at $20 \mathrm{~K}$ and under VUV irradiation, the acetonitrile can lead to the amino acetonitrile formation. These results suggest that acetonitrile and amino acetonitrile can be formed in astrophysical environments that are submitted to VUV irradiations.
\end{abstract}

Key words. astrochemistry - ISM: molecules - methods: laboratory

\section{Introduction}

The evolution of labile and functionalized organic matter starts in dense molecular clouds. These cold (10-50 K) clouds are composed of interstellar grains that show large amounts of dirty ices in astronomical infrared vibrational spectra, which are mainly composed of water but also of other molecules such as $\mathrm{CO}, \mathrm{CO}_{2}, \mathrm{CH}_{3} \mathrm{OH}, \mathrm{NH}_{3}$, etc. (Dartois 2005). During the grain evolution, this material will undergo numerous chemical modifications caused by ionic and VUV irradiations as well as thermal effects. In the laboratory, where templates can be studied more precisely, this evolution ultimately leads to the formation of a complex semi-refractory organic residue that may well exist in molecular clouds and in the early phases of planetary systems formation. Areas of the initial molecular cloud collapse to form in proto-stars, which evolve into solar nebulae that potentially lead to a planetary system like ours. During this evolution, interstellar grains agglomerate and lead to small objects such as comets or asteroids that include organic matter, some of which may then have been preserved from their original InterStellar Medium (ISM) state. Later on, these small bodies may serve as reservoirs of organic material for the development of a prebiotic chemistry at the surface of telluric planets. Thereby, the understanding of the constitution and of the molecular evolution of interstellar matter provides clues about the organic matter that has been delivered onto the primitive Earth or onto any telluric planet. In this contribution, we experimentally investigate the formation of several nitrile derivatives in environments relevant to interstellar ices or comets. Nitriles are compounds of interest in astrochemistry because they are easily detected in the gas phase in interstellar and planetary media (Belloche et al. 2008; Olmi et al. 1993; Remijan et al. 2004; Whittet et al. 2001). Nitrile derivatives are considered as potential amino acid precursors in astrophysical environments (Bernstein et al. 2002; Elsila et al. 2007; Hudson et al. 2008). However, amino acids have not been detected in the ISM, which may support experimental results showing that nitriles are more stable under VUV irradiation than their amino acids homologs (Bernstein et al. 2004). In addition, amino acid formation from nitriles requires an acid hydrolysis step that is not possible in interstellar grains (Rimola et al. 2010). Thus, their relevance as biomolecule precursors in prebiotic chemistry have very probably been important after their delivery onto the primitive Earth through an early bombardment by comets or asteroids (Oro 1961). We therefore focused our investigation on the smallest alkyl nitriles detected in astrophysical environments, acetonitrile and amino acetonitrile. Acetonitrile 
$\left(\mathrm{CH}_{3} \mathrm{CN}\right)$ was detected in comets (Huebner et al. 1974; Snyder \& Buhl 1971), Titan's atmosphere (Lara et al. 1996), in hot cores (Belloche et al. 2009; Remijan et al. 2004) and in protostellar objects (Codella et al. 2009). Experiments simulating interstellar ices have suggested that in some conditions, acetonitrile could be considered as an amino acid precursor (Hudson et al. 2008). Indeed, when a refractory residue that comes from an irradiated ice mixture of acetonitrile and water is hydrolysed under acidic conditions, several amino acids are detected. However, acetonitrile has not been detected in the solid phase of the interstellar medium and its formation pathway is not yet explained. Experiments suggest that acetonitrile could be formed in the ISM from the photochemistry of acetamide, which is detected in hot cores (Hollis et al. 2006), and whose photodegradation leads to acetonitrile and water in the gas or solid phases (Duvernay et al. 2007; Spall \& Steaciel 1957).

Amino acetonitrile $\left(\mathrm{NH}_{2} \mathrm{CH}_{2} \mathrm{CN}\right)$ has been observed with radio astronomy in the hot core Sgr B2(N) (Belloche et al. 2008), but not in the solid phase. It is considered as a potential glycine precursor through the Strecker reaction in extraterrestrial objects such as in meteorites and comets (Bernstein et al. 2002; Elsila et al. 2007; Elsila et al. 2009; Koch et al. 2008). Only theoretical studies were done on the formation of amino acetonitrile. Some authors have proposed that it could be formed at the surface of interstellar dust grains or in the gas phase from methanimine and hydrogen cyanide or hydrogen isocyanide in a water environment (Basiuk et al. 2002a,b; Koch et al. 2008; Woon 2002; Xu $\&$ Wang 2007). The purpose of this work is to present the first experimental approach for the formation of acetonitrile and amino acetonitrile in interstellar-ice-like conditions. We test here the possibility to form acetonitrile from one of the simplest amines, ethylamine $\left(\mathrm{C}_{2} \mathrm{H}_{5} \mathrm{NH}_{2}\right)$, under VUV irradiation at $20 \mathrm{~K}$ and the formation of amino acetonitrile from a mixture of acetonitrile and ammonia under VUV irradiation at $20 \mathrm{~K}$. We present the astrophysical implications of our results in Sect. 3.3.

\section{Experimental details}

For this study, the following reagents were used: Acetonitrile (Acros Organics, 99\% purity), acetonitrile ${ }^{15} \mathrm{~N}$ (Sigma Aldrich, 98\% purity), amino acetonitrile (Sigma Aldrich, 97\% purity), ethylamine (Fluka, 99\% purity), ethylamine ${ }^{15} \mathrm{~N}$ (Isotech Aldrich, $98 \%$ purity) and ammonia (Air Liquide, $99.9 \%$ purity). Reagents were mixed in different ratios in a pyrex line using standard manometric techniques. Liquid reagents (acetonitrile, aminoacetonitrile) were vacuum transferred away from their liquid state by evaporation and if necessary, their resulting vapors were mixed in a glass bulb with other reagents. The gaseous mixture was deposited at a $6 \times 10^{-1} \mathrm{~mol} \mathrm{~min}^{-1}$ rate on a gold platted surface kept at $20 \mathrm{~K}$ with the help of a model $21 \mathrm{CTI}$ cold head. The warming up of the sample was realized at a $4 \mathrm{~K} \mathrm{~min}^{-1}$ heating rate using a resistive heater along with a Lakeshore model 331 temperature controller. The VUV irradiation $(\lambda>120 \mathrm{~nm})$ was realized using a microwave discharge hydrogen flow lamp (Opthos Instruments). The flux of photons is estimated to be in the range $10^{14}-10^{15}$ photons $\mathrm{cm}^{-2} \mathrm{~s}^{-1}$. The infrared spectra of the samples were recorded in a reflection mode between 4000 and $600 \mathrm{~cm}^{-1}$ using a Nicolet Magna 750 FTIR spectrometer with a MCT detector. Each spectrum was averaged over one hundred scans and has a $1 \mathrm{~cm}^{-1}$ resolution. The mass spectra were monitored using a RGA quadrupole mass spectrometer (MKS Microvision-IP plus) while the products are desorbed during the controlled temperature ramp. The ionisation source is a $70 \mathrm{eV}$ impact electronic source and the mass spectra are recorded

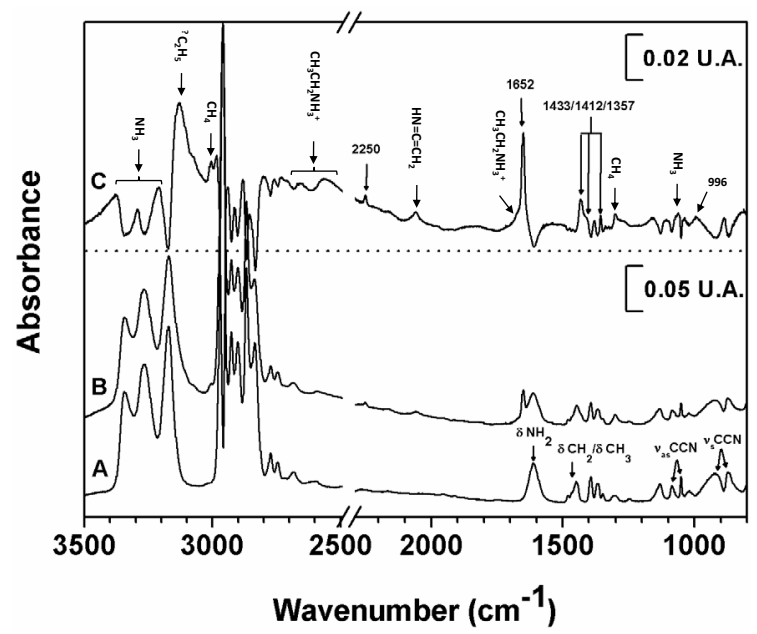

Fig. 1. Infrared spectra of pure ethylamine ice (A), pure ethylamine after $240 \mathrm{~min}$ of VUV irradiation at $20 \mathrm{~K}$ (B) and difference spectrum (C) of (A) and (B). All spectra were recorded at $20 \mathrm{~K}$.

between 1 and $200 \mathrm{amu}$. The photochemical yield of the photoproducts was obtained with the method already described in Bossa et al. (2009a).

\section{Results and discussion}

\subsection{From ethylamine to acetonitrile through photolysis}

In this section, we investigate the formation of acetonitrile from the photolysis of ethylamine. The infrared spectra of pure ethylamine before (Fig. 1A) and after $240 \mathrm{~min}$ of irradiation (Fig. 1B) at a temperature of $20 \mathrm{~K}$ are displayed in Fig. 1 along with vibrational assignments. Solid ethylamine can be trapped at low temperature under trans- and left-conformers as shown by the presence of the two bands at $1084 \mathrm{~cm}^{-1}$, and $1150 \mathrm{~cm}^{-1}$ which are related to the CCN stretching modes (Durig et al. 2006; Zeroka et al. 1999). The comparison of the two spectra in Figs. 1A and B shows the appearance of new infra-red bands. The new features of these photoproducts can be more easily observed by subtracting those two spectra (Fig. 1C).

The most intense band appears at $1652 \mathrm{~cm}^{-1}$. This band corresponds to the $\mathrm{C}=\mathrm{N}$ or $\mathrm{C}=\mathrm{C}$ stretching mode. The presence of an unsaturated compound is consistent with the elimination of molecular hydrogen $\left(\mathrm{H}_{2}\right)$ induced by ultra-violet photons. But at this stage, from the $1652 \mathrm{~cm}^{-1}$ band alone, we cannot conclude about the photoproduct formed during this process since this molecular hydrogen could be lost as well from the C-N bond to give ethanimine (Hashiguchi et al. 1984; Stolkini et al. 1977) or from the $\mathrm{C}-\mathrm{C}$ bond to produce ethenamine (Hamada et al. 1984) (Eqs. (1) and (2)). These two photoproducts have, unfortunately, a very close absorption band in this region.

$$
\begin{aligned}
& \mathrm{CH}_{3} \mathrm{CH}_{2} \mathrm{NH}_{2}+h v \rightarrow \mathrm{CH}_{3} \mathrm{CH}=\mathrm{NH}+\mathrm{H}_{2} \\
& \mathrm{CH}_{3} \mathrm{CH}_{2} \mathrm{NH}_{2}+h v \rightarrow \mathrm{CH}_{2}=\mathrm{CHNH}_{2}+\mathrm{H}_{2} .
\end{aligned}
$$

Next to the $1652 \mathrm{~cm}^{-1}$ band, some other bands can be assigned to ethanimine. The bands at $1433 \mathrm{~cm}^{-1}$ and $1412 \mathrm{~cm}^{-1}$ are close to the anti-symmetric and symmetric modes of the methyl deformation in ethanimine $\left(\mathrm{CH}_{3} \mathrm{CH}=\mathrm{NH}\right)$ (Stolkini et al. 1977). Other bands at $1357 \mathrm{~cm}^{-1}$ and between $1100-1000 \mathrm{~cm}^{-1}$ could also be consistent with the mixed and the deformation modes of this species (Stolkini et al. 1977; Hashiguchi et al. 1984). For removing any ambiguity with these assignments, the isotopomer 
Table 1. Positions and attributions of infrared absorption bands after a 240 min photolysis at $20 \mathrm{~K}$ of a pure ethylamine $\left(\mathrm{C}_{2} \mathrm{H}_{5} \mathrm{NH}_{2}\right)$ ice and of its pure isotopomer $\left(\mathrm{C}_{2} \mathrm{H}_{5}{ }^{15} \mathrm{NH}_{2}\right)$.

\begin{tabular}{|c|c|c|c|c|}
\hline $\begin{array}{l}\text { Ice } \\
(\lambda>120 \mathrm{~nm})\end{array}$ & $\begin{array}{c}\text { Wavenumber } \\
\left(\mathrm{cm}^{-1}\right)\end{array}$ & Attribution & Identification & Ref. \\
\hline $\begin{array}{l}\mathrm{C}_{2} \mathrm{H}_{5} \mathrm{NH}_{2} \\
\text { (pure, 20 K) }\end{array}$ & $\begin{array}{l}3380 \\
3294 \\
3209 \\
3127 \\
3008 \\
2655 \\
2566 \\
2250 \\
2165 \\
2080 \\
2058 \\
1670 \\
1652 \\
1433 \\
1412 \\
1357 \\
1301 \\
1062 \\
996 \\
\end{array}$ & $\begin{array}{c}\mathrm{NH}_{3} \\
\mathrm{NH}_{3} \\
\mathrm{NH}_{3} \\
\mathrm{C}_{2} \mathrm{H}_{5} \\
\mathrm{CH}_{4} \\
{\left[\mathrm{CH}_{3} \mathrm{CH}_{2} \mathrm{NH}_{3}^{+}\right]} \\
{\left[\mathrm{CH}_{3} \mathrm{CH}_{2} \mathrm{NH}_{3}^{+}\right]} \\
\mathrm{CH}_{3} \mathrm{C} \equiv \mathrm{N} \\
\mathrm{CH}_{3}-\mathrm{NC} ? \\
{[\mathrm{CN}]} \\
\mathrm{CH}_{2}=\mathrm{C}=\mathrm{NH} \\
{\left[\mathrm{CH}_{3} \mathrm{CH} \mathrm{NH}_{3}^{+}\right]} \\
\mathrm{CH}_{3} \mathrm{CH}=\mathrm{NH} \\
\mathrm{CH}_{3} \mathrm{CH}=\mathrm{NH} \\
\mathrm{CH}_{3} \mathrm{CH}=\mathrm{NH} \\
\mathrm{CH}_{3} \mathrm{CH}=\mathrm{NH} \\
\mathrm{CH} \\
\mathrm{NH}_{3} \\
\mathrm{CH}_{2}=\mathrm{C}=\mathrm{NH}\end{array}$ & $\begin{array}{c}\left(v_{3}\right)\left(v_{\mathrm{as}}\right) \mathrm{NH}_{3} \\
\left(2 v_{4}\right) \text { harmonic } \\
\left(v_{1}\right)\left(v_{\mathrm{s}}\right) \mathrm{NH}_{3} \\
v_{\mathrm{as}} \mathrm{CH}_{2} \\
v \mathrm{CH}_{4} \\
\text { Fermi res. } \\
\text { Fermi res. } \\
v \mathrm{C} \equiv \mathrm{N} \\
v \mathrm{NC} ? \\
v \mathrm{C}-\mathrm{N} \\
v_{\mathrm{as}} \mathrm{C}=\mathrm{C}=\mathrm{N} \\
\delta_{\mathrm{as}} \mathrm{NH}_{3}^{+} \\
v \mathrm{C}=\mathrm{N} \\
\delta_{\mathrm{as}} \mathrm{CH}_{3} \\
\delta_{\mathrm{s}} \mathrm{CH}_{3} \\
\text { mixed mode } \\
\text { dd } \mathrm{CH}_{4} \\
\left(v_{2}\right) \text { umbrella mode } \\
\delta \mathrm{CNH}\end{array}$ & $\begin{array}{c}\text { (d'Hendecourt et al. 1986; Ferraro et al. 1980) } \\
\text { (d'Hendecourt et al. 1986; Ferraro et al. 1980) } \\
\text { (d'Hendecourt et al. 1986; Ferraro et al. 1980) } \\
\text { (Sogoshi et al. 1997) } \\
\text { (d'Hendecourt et al. 1986; Gerakines et al. 1996) } \\
\text { (Hagemann \& Bill 1984) } \\
\text { (Hagemann \& Bill 1984) } \\
\text { (d'Hendecourt et al. 1986; Hudson \& Moore 2004) } \\
\text { (Hudson \& Moore 2004) } \\
\text { (Gerakines et al. 2004; Moore \& Hudson 2003) } \\
\text { (Hudson \& Moore 2004; Jacox \& Milligan 1963) } \\
\text { (Hagemann \& Bill 1984; Zeroka et al. 1999) } \\
\text { (Hashiguchi et al. 1984; Stolkini et al. 1977) } \\
\text { (Stolkini et al. 1977) } \\
\text { (Stolkini et al. 1977) } \\
\text { (Hashiguchi et al. 1984; Stolkini et al. 1977) } \\
\text { (d'Hendecourt et al. 1986; Gerakines et al. 1996) } \\
\text { (d'Hendecourt et al. 1986; Ferraro et al. 1980) } \\
\text { (Jacox \& Milligan 1963) }\end{array}$ \\
\hline $\begin{array}{l}\mathrm{C}_{2} \mathrm{H}_{5}{ }^{15} \mathrm{NH}_{2} \\
\text { (pure, } 20 \mathrm{~K} \text { ) }\end{array}$ & $\begin{array}{l}3202 \\
2224 \\
2136 \\
2071 \\
2031 \\
1634 \\
1433 \\
1412 \\
\end{array}$ & $\begin{array}{c}{ }^{15} \mathrm{NH}_{3} \\
\mathrm{CH}_{3} \mathrm{C} \equiv{ }^{15} \mathrm{~N} \\
\mathrm{CH}_{3}-{ }^{15} \mathrm{NC} ? \\
{\left[\mathrm{C}^{15} \mathrm{~N}\right]} \\
\mathrm{CH}_{2}=\mathrm{C}={ }^{15} \mathrm{NH} \\
\mathrm{CH}_{3} \mathrm{CH}={ }^{15} \mathrm{NH} \\
\mathrm{CH}_{3} \mathrm{CH}={ }^{15} \mathrm{NH} \\
\mathrm{CH}_{3} \mathrm{CH}={ }^{15} \mathrm{NH} \\
\end{array}$ & $\begin{array}{c}\left(v_{1}\right)\left(v_{\mathrm{s}}\right)^{15} \mathrm{NH}_{3} \\
v \mathrm{C} \equiv{ }^{15} \mathrm{~N} \\
v{ }^{15} \mathrm{NC} \\
v \mathrm{C}-{ }^{15} \mathrm{~N} \\
v \mathrm{C}={ }^{15} \mathrm{~N} \\
v \mathrm{C}={ }^{15} \mathrm{~N} \\
\delta_{\mathrm{as}} \mathrm{CH}_{3} \\
\delta_{\mathrm{s}} \mathrm{CH}_{3}\end{array}$ & (Gerakines et al. 2004; Moore \& Hudson 2003) \\
\hline
\end{tabular}

Notes. Vibration mode: stretching $(v)$, bending $(\delta)$, rocking $(\rho)$, torsion $(\tau)$, wagging $($ w). Type of vibration mode: asymmetric (as), symmetric $(\mathrm{s})$.

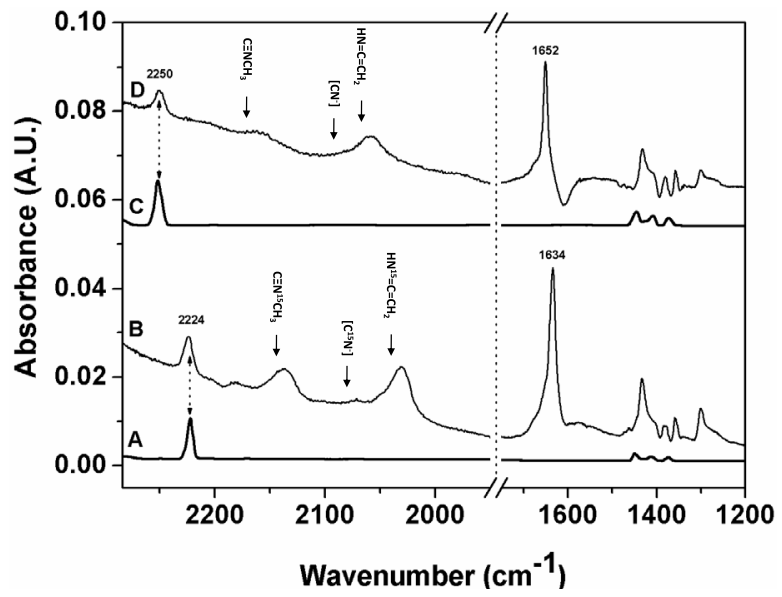

Fig. 2. Infrared spectra of pure acetonitrile ${ }^{15} \mathrm{~N}$ ice (A) and difference spectrum between pure ethylamine ${ }^{15} \mathrm{~N}$ and ethylamine ${ }^{15} \mathrm{~N}$ after 240 min of VUV irradiation at $20 \mathrm{~K}$ (B). Spectra (C) and (D) are identical to (A) and (B) with the isotopomer ${ }^{14} \mathrm{~N}$. All spectra were recorded at $20 \mathrm{~K}$.

${ }^{15} \mathrm{~N}$ photolysis of ethylamine at $20 \mathrm{~K}$ was compared to the nor-
mal isotopomer (Fig. 2 ). This comparison shows a clear shift of
the wavenumber attributed to $\mathrm{C}=\mathrm{N}$ vibration, which shifts from
$1652 \mathrm{~cm}^{-1}$ for ${ }^{14} \mathrm{~N}$ to $1634 \mathrm{~cm}^{-1}$ for ${ }^{15} \mathrm{~N}$. This variation is consis-
tent with the presence of the nitrogen in the $\mathrm{C}=\mathrm{N}$ group because
of the increase of the reduced mass of the vibrator. Furthermore, the methyl deformations at $1433 \mathrm{~cm}^{-1}$ and $1412 \mathrm{~cm}^{-1}$ are not shifted in the ${ }^{15} \mathrm{~N}$ photolysis. It appears from these results that the primary product formed during the photolysis of ethylamine is ethanimine $\left(\mathrm{CH}_{3} \mathrm{CH}=\mathrm{NH}\right)$.

Another interesting feature is the band at $2250 \mathrm{~cm}^{-1}$ (Fig. 1C and Table 1). This band is characteristic of a $\mathrm{C} \equiv \mathrm{N}$ stretching mode in an aliphatic nitrile. To check if this band could correspond to a vibration mode of acetonitrile, the smallest nitrile that could be formed in these conditions, the spectrum of solid acetonitrile, was compared to the photolysis spectra of solid ethylamine at $20 \mathrm{~K}$ (Fig. 2). Evidently the band due to the $\mathrm{C} \equiv \mathrm{N}$ stretching mode of acetonitrile $\left(2250 \mathrm{~cm}^{-1}\right)$ has the same position as the nitrile band observed on the photolysis spectrum (Figs. 2C and D). This implies that acetonitrile is present after the photolysis of a pure ice of ethylamine. To strengthen this identification, the spectrum of the $\mathrm{CH}_{3} \mathrm{C} \equiv{ }^{15} \mathrm{~N}$ acetonitrile isotopomer was also compared to the one obtained after photolysis of ethylamine ${ }^{15} \mathrm{~N}$ (Figs. 2A and B). The band at $2250 \mathrm{~cm}^{-1}$ is not observed in the ${ }^{15} \mathrm{~N}$ spectrum recorded after VUV photolysis, which is consistent with the attribution to a nitrile function. Moreover, a new band is present at $2224 \mathrm{~cm}^{-1}$. This band has the same position as the one of the stretching vibration mode of $\mathrm{C} \equiv{ }^{15} \mathrm{~N}$ in $\mathrm{CH}_{3} \mathrm{C} \equiv{ }^{15} \mathrm{~N}$. From these results, we can conclude that acetonitrile is formed during the photolysis of ethylamine ice at $20 \mathrm{~K}$. The photochemical yield of the acetonitrile formation is estimated from the amount of ethylamine consumed and the amount of acetonitrile observed after irradiation. We used the band strengths of the methylamine: $\mathrm{NH}_{2}(A=4.3 \times$ $\left.10^{-18} \mathrm{~cm} \mathrm{molecule}^{-1}\right)$ and $\mathrm{CH}_{3}\left(A=1.5 \times 10^{-18} \mathrm{~cm}\right.$ molecule $\left.{ }^{-1}\right)$ 


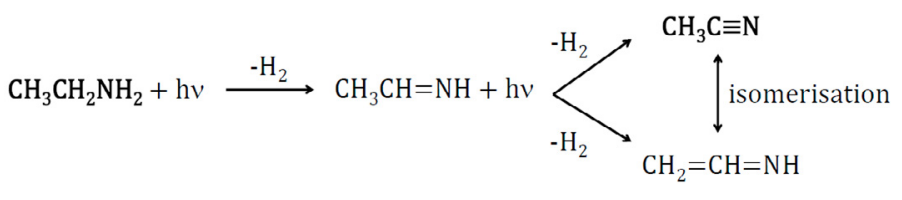

Fig. 3. Pathways for acetonitrile formation from ethylamine.

vibrators (Holtom et al. 2005), at $1612 \mathrm{~cm}^{-1}$ and at $1131 \mathrm{~cm}^{-1}$ respectively to determine the amount of ethylamine consumed after VUV irradiation because the corresponding values are not known for this compound. Using the above approximation and the column density of acetonitrile at $2250 \mathrm{~cm}^{-1}(A=2.2 \times$ $10^{-18} \mathrm{~cm} \mathrm{molecule}^{-1}$ ) (d'Hendecourt et al. 1986), the branching ratio of formed acetonitrile/consumed ethylamine is estimated to be around $32 \%$ at $20 \mathrm{~K}$ and after $240 \mathrm{~min}$ of VUV irradiation. Given that $14 \%$ of the ethylamine was consumed, the photochemical yield of acetonitrile is estimated to be about 4\%. Other molecular compounds were identified and are listed in Table 1, and displayed in Figs. 1 and 2. Among them, we note isoacetonitrile $\left(\mathrm{CH}_{3} \mathrm{~N} \equiv \mathrm{C}\right)$ because the $\mathrm{N} \equiv \mathrm{C}$ vibrator of this isonitrile can be assigned to the $2165 \mathrm{~cm}^{-1}$ feature (Fig. 2D) (Hudson Moore 2004), which is confirmed by the ${ }^{15} \mathrm{~N}$ experiment (Fig. 2B). This compound is known for being an acetonitrile photoproduct formed by acetonitrile isomerisation. Another interesting species is ketenimine $\left(\mathrm{CH}_{2}=\mathrm{C}=\mathrm{NH}\right)$, which is identified by the bands at $2058 \mathrm{~cm}^{-1}$ and $996 \mathrm{~cm}^{-1}$ (Figs. 2B and D) (Hudson \& Moore 2004; Jacox \& Milligan 1963).

Ketenimine could be formed from the acetonitrile isomerisation or from the dehydrogenation of the ethanimine. The photochemical yield of formation of ketemine and isoacetonitrile were estimated using the band strengths given by Hudson et al. (Hudson \& Moore 2004). The corresponding yields are $0.4 \%$, and $1.3 \%$ respectively. Unfortunately, we could not estimate the photochemical yield for the formation of ethanimine because we lacked the band strength for this compound. Methane was also detected from the bands at $3008 \mathrm{~cm}^{-1}$ and $1301 \mathrm{~cm}^{-1}$, which remain unchanged in frequency after the VUV photolysis of $\mathrm{C}_{2} \mathrm{H}_{5}{ }^{15} \mathrm{NH}_{2}$. This methane formation could be induced by the acetonitrile photolysis through the reaction (Hudson \& Moore 2004):

$$
\mathrm{CH}_{3} \mathrm{C} \equiv \mathrm{N}+h v \rightarrow \mathrm{CH}_{4}+\mathrm{HCN} \text {. }
$$

Cyanhydric acid is not detected since it probably reacts with the excess of ethylamine to give the following salt $\left[\mathrm{CH}_{3} \mathrm{CH}_{2} \mathrm{NH}_{3}^{+}\right.$ $\mathrm{CN}^{-}$], which is confirmed by the band at $2655 \mathrm{~cm}^{-1}$ and at $1670 \mathrm{~cm}^{-1}$ consistent with the ion $\mathrm{CH}_{3} \mathrm{CH}_{2} \mathrm{NH}_{3}^{+}$(Hagemann \& Bill 1984) and the shoulder at $2080 \mathrm{~cm}^{-1}$ consistent with the $\mathrm{CN}^{-}$ion (Gerakines et al. 2004; Moore \& Hudson 2003). Finally, ammonia was identified from the infrared bands at $3380 \mathrm{~cm}^{-1}, 3294 \mathrm{~cm}^{-1}, 3209 \mathrm{~cm}^{-1}$ and $1062 \mathrm{~cm}^{-1}$. Ammonia was formed from the reaction between ${ }^{\circ} \mathrm{NH}_{2}$ and ${ }^{\circ} \mathrm{H}$ coming from ethylamine photodissociation. This is also confirmed by the presence of the radical $\mathrm{C}_{2} \mathrm{H}_{5}$, identified by the band at $3127 \mathrm{~cm}^{-1}$ (Sogoshi et al. 1997).

From these results, we can conclude that photochemistry of a pure ice at $20 \mathrm{~K}$ leads to the formation of acetonitrile. The process involves a first elimination of molecular hydrogen that leads to ethanimine. Then, from ethanimine a second elimination of molecular hydrogen probably leads to acetonitrile formation. However, we cannot exclude that acetonitrile could be formed by ketenimine isomerisation, which can also be synthesized from ethanamine by elimination of molecular hydrogen (Fig. 3).

\subsection{Amino acetonitrile synthesis from photochemistry of an acetonitrile/ammonia mixture}

In this section, we take a closer look at the amino acetonitrile formation from photochemical reactions of an acetonitrile/ammonia ice mixture. The VUV photochemistry $(\lambda>$ $120 \mathrm{~nm}$ ) of a pure solid of acetonitrile has already been investigated (12 to $25 \mathrm{~K}$ ) (Hudson \& Moore 2004). Hudson et al. (2004) have shown, as we did in the previous section, three main photoproducts: ketenimine $\left(\mathrm{CH}_{2}=\mathrm{C}=\mathrm{NH}\right)$, isoacetonitrile $\left(\mathrm{CH}_{3}-\right.$ $\mathrm{N} \equiv \mathrm{C}$ ) and hydrogen cyanide with methane. Ketenimine is one of the major compounds formed during photolysis, which results from an intramolecular rearrangement (Hudson \& Moore 2004) through the pathway.

$\mathrm{CH}_{3} \mathrm{C} \equiv \mathrm{N}+h v \rightarrow\left[{ }^{\cdot} \mathrm{CH}_{2}-\mathrm{C} \equiv \mathrm{N}+{ }^{\cdot} \mathrm{H}\right] \rightarrow \mathrm{CH}_{2}=\mathrm{C}=\mathrm{NH}$.

The radical intermediate $\mathrm{CH}_{2}-\mathrm{CN}$ could be a useful starting point to form the amino acetonitrile because, it could react with the $\mathrm{NH}_{2}$ radical coming from ammonia photolysis,

$\mathrm{CH}_{3} \mathrm{C} \equiv \mathrm{N}+\mathrm{NH}_{3}+h v \rightarrow\left[{ }^{\circ} \mathrm{CH}_{2}-\mathrm{C} \equiv \mathrm{N}+{ }^{\circ} \mathrm{NH}_{2}+2 \mathrm{H}^{\cdot}\right]$

$\left[{ }^{\circ} \mathrm{CH}_{2}-\mathrm{C} \equiv \mathrm{N}+{ }^{\circ} \mathrm{NH}_{2}+2 \mathrm{H}^{\cdot}\right] \rightarrow \mathrm{NH}_{2}-\mathrm{CH}_{2}-\mathrm{C} \equiv \mathrm{N}+\mathrm{H}_{2}$.

In order to test this hypothesis, a mixture of acetonitrile/ammonia was photolysed with VUV photons $(\lambda>120 \mathrm{~nm})$. A comparison between an infrared spectrum of amino acetonitrile and a spectrum of an acetonitrile/ammonia mixture rapidly shows that infrared spectroscopy is not the most easy and convenient method to identify the formation of amino acetonitrile. Indeed, the $\mathrm{NH}_{2}$ vibration modes of ammonia hide those of amino acetonitrile, while the $\mathrm{C} \equiv \mathrm{N}$ vibration mode of acetonitrile masks the amino acetonitriles one. However, the sublimation temperature provides a way to distinguish between acetonitrile and amino acetonitrile, since the acetonitrile sublimation occurs at around $158 \mathrm{~K}$, while that of amino acetonitrile occurs at higher temperatures, at around $200 \mathrm{~K}$. Unfortunately, infrared spectroscopy is not sensitive enough to provide a secure detection of amino acetonitrile. The only way to follow the amino acetonitrile formation is to use a mass spectrometer. Consequently, the characterization of amino acetonitrile formed during the photolysis of an acetonitrile/ammonia mixture was conducted by monitoring the partial pressure of the ion at $\mathrm{m} / \mathrm{z} 55$ and 56 as a function of temperature.

This is shown in Figs. 4A and B, which display the mass spectra of acetonitrile and that of amino acetonitrile respectively. This last one can be identified without any ambiguity by its molecular ion at $m / z 56$ and the fragment ion $m / z 55$ (Fig. 4B). An ice mixture composed of acetonitrile/ammonia with a 5:1 ratio was photolysed $(\lambda>120 \mathrm{~nm})$ during $190 \mathrm{~min}$ at $20 \mathrm{~K}$. After this photolysis, the mixture was warmed from $20 \mathrm{~K}$ to $300 \mathrm{~K}$ at $4 \mathrm{~K} \mathrm{~min}^{-1}$ while the partial pressure of the ions at $\mathrm{m} / \mathrm{z} 56$ (Fig. 5A) and $\mathrm{m} / \mathrm{z} 55$ (Fig. 5B) was recorded. Figure 5 displays the evolution of the partial pressures of the mentioned ions.

The spectrum profiles obtained after irradiation (Figs. 5A and B) were also compared with the spectrum of the same ions resulting in the warming up of the same acetonitrile/ammonia mixture without VUV irradiation (Figs. 5C and D). For the irradiated sample (Figs. 5A and B), above $190 \mathrm{~K}$, the partial pressure of the ion at $m / z 55$ increases to a maximum at $205 \mathrm{~K}$ and slows down to reach the baseline at $220 \mathrm{~K}$, whereas with the non-irradiated sample the profile stays flat. The same profile is observed for the ion at $m / z$ 56. Even if this variation is weak compared to the noise, these two intensities rise in the temperature range $190-220 \mathrm{~K}$ associated to a higher intensity for the 

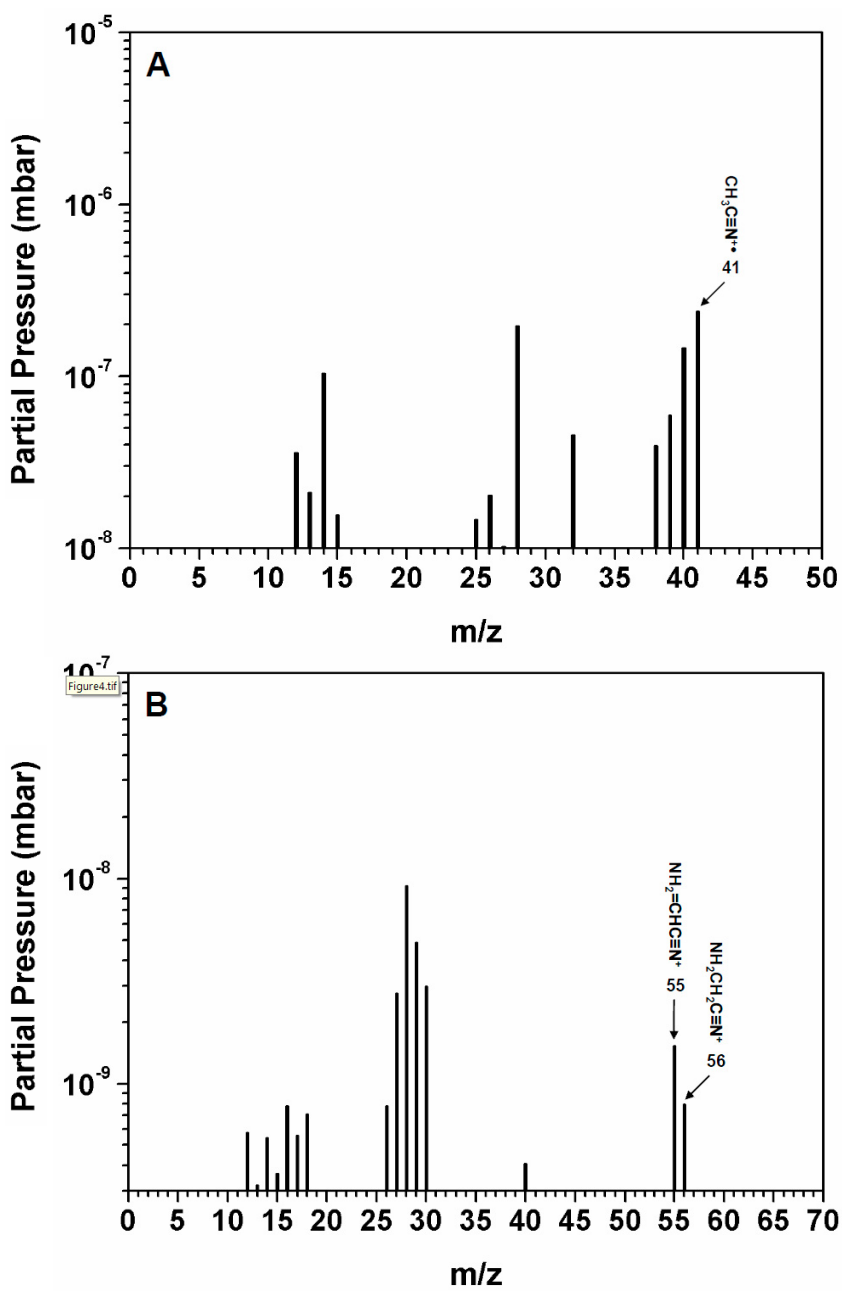

Fig. 4. Mass spectrum of evaporating acetonitrile at $158 \mathrm{~K}$ after warming up of the pure ice of this molecule (electronic ionization $70 \mathrm{eV}$ ) (A) and evaporating amino acetonitrile at $200 \mathrm{~K}$ after warming up of the pure ice of this molecule (electronic ionization $70 \mathrm{eV}$ ) (B).

$m / z 55$ ion compared with the ion of $m / z, 56$ ion (Figs. 4A and B), which confirms the sublimation of amino acetonitrile.The signal at $m / z, 55$ is then attributed to the $\mathrm{H}_{2} \mathrm{~N}=\mathrm{CHC} \equiv \mathrm{N}^{+}$fragment ion, which comes from the fragmentation of the $\mathrm{H}_{2} \mathrm{NCH}_{2} \mathrm{C} \equiv \mathrm{N}^{+}$. molecular ion represented by the signal at $m / z 56$.

\subsection{Astrophysical discussion}

All species used in our experiments have been detected in extraterrestrial environments in either the gas or in the solid phases. In particular, ethylamine has been identified in the cometexposed materials from stardust (Glavin et al. 2008). Different pathways for ethylamine formation were already suggested for these environments. For example, the formation of ethylamine in the gas phase from a neutral-neutral reaction between the methyl radical and the methanimine followed by hydrogenation of the resulting product was proposed for its formation at the surface of interstellar or cometary grains (Bernstein et al. 1995). Other authors proposed ethylamine formation from the photochemistry of a mixture of ethylene and ammonia. Ethylene can be formed from methane (Atreya 1992; Gerakines et al. 1996) (Eqs. (7) and (8)) which represents $1 \%$ to $4 \%$ (with respect to water) of the composition of interstellar ices (Dartois 2005),

$2 \mathrm{CH}_{4}+h v \rightarrow 2 \mathrm{CH}_{3}^{\cdot}+2 \mathrm{H}^{\cdot} \rightarrow \mathrm{C}_{2} \mathrm{H}_{6}+\mathrm{H}_{2}$

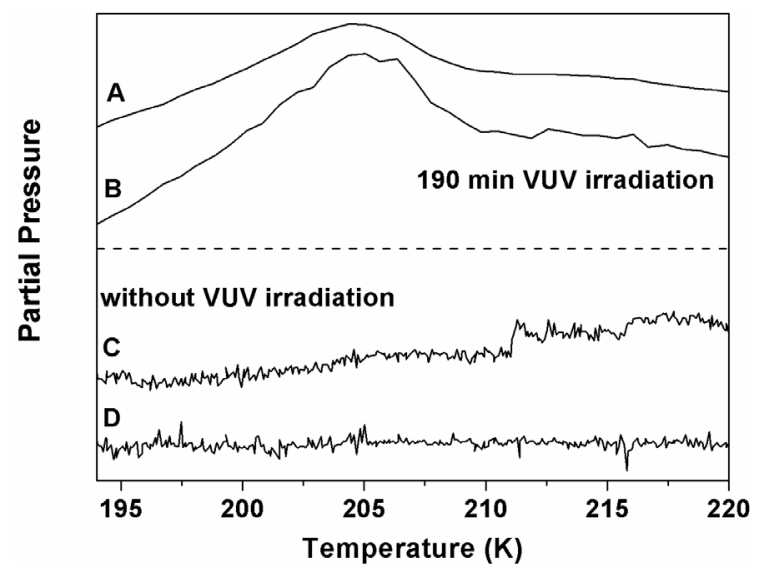

Fig. 5. Partial pressure for ions $m / z, 56$ (A and C) and 55 (B and D) as a function of temperature during the warm-up of an ice mixture of acetonitrile:ammonia (5:1) (A and B) previously irradiated at $20 \mathrm{~K}$ during 190 min or (C and D) without irradiation.

$\mathrm{C}_{2} \mathrm{H}_{6}+\mathrm{H}_{2}+h v \rightarrow \mathrm{C}_{2} \mathrm{H}_{4}+\mathrm{H}_{2}$.

Then, ammonia is dissociated to give the aminyl radical ${ }^{\circ} \mathrm{NH}_{2}$ (Gerakines et al. 1996) (Eq. (9)), which reacts further with the ethyl radical that can originate from the partial hydrogenation of ethylene (Eq. (10)) or by the partial photolysis of ethane (Eq. (11)) to yield ethylamine (Eq. (12)),

$$
\begin{aligned}
& \mathrm{NH}_{3}+h v \rightarrow \mathrm{NH}_{2}+{ }^{\cdot} \mathrm{H} \\
& \mathrm{C}_{2} \mathrm{H}_{4}+{ }^{\cdot} \mathrm{H} \rightarrow{ }^{\cdot} \mathrm{C}_{2} \mathrm{H}_{5} \\
& \mathrm{C}_{2} \mathrm{H}_{6}+h v \rightarrow{ }^{\cdot} \mathrm{C}_{2} \mathrm{H}_{5}+{ }^{\cdot} \mathrm{H} \\
& \mathrm{C}_{2} \mathrm{H}_{5}+{ }^{\cdot} \mathrm{NH}_{2} \rightarrow \mathrm{C}_{2} \mathrm{H}_{5} \mathrm{NH}_{2} .
\end{aligned}
$$

Once formed, ethylamine can adsorb on the surface of interstellar or cometary grains, where it will undergo solid phase reactivity. Its presence in interstellar ices is strengthened by its detection in volatile compounds coming from cometary surface (Glavin et al. 2008). Consequently, by taking into account the above arguments and the results obtained in this work, we suggest that acetonitrile can be synthesized at the surface of interstellar or cometary grains following a two step dehydrogenation of ethylamine under VUV irradiation. In pure ethylamine the photochemical yield of acetonitrile after $240 \mathrm{~min}$ is $4 \%$. This yield in acetonitrile needs to be lowered in real astrophysical environments, because the proportion of water could shield ethylamine from VUV photons, which should decrease this yield. Additionally, from recent experimental results, we can draw up another acetonitrile formation pathway. Our group have shown that it is possible to form in the solid phase amino alcohols $\left(\mathrm{NH}_{2} \mathrm{CH}(\mathrm{R}) \mathrm{OH}\right)$ with the first step of the Strecker synthesis from the thermal reaction between an aldehyde or a ketone and ammonia (Bossa et al. 2009b; Duvernay et al. 2010). From the specific reaction between acetaldehyde and ammonia, the amino ethanol was synthesized and its VUV irradiation leads to acetamide. Acetamide under VUV irradiation then provides acetonitrile and water (Duvernay et al. 2007). Once formed, acetonitrile could be a precursor of another nitrile, the amino acetonitrile. Since at the surface of grains acetonitrile can co-exist with ammonia, which represents up to $10 \%$ (upper limit relative to water) (Dartois \& d'Hendecourt 2001) of the interstellar ice composition, it will react through a photochemical reaction leading to amino acetonitrile. Others ways of synthesis of amino acetonitrile have been proposed through theoretical studies. Some authors suggest a 
reaction similar to the third step of the Strecker reaction between methanimine and hydrogen cyanide or hydrogen isocyanide at the surface of interstellar grains (Koch et al. 2008), where the methanimine could be formed from the hydrogenation of hydrogen cyanide or from the dehydrogenation of methylamine.

\section{Conclusion}

In this contribution, we investigated the formation of two nitrile derivatives, acetonitrile and amino acetonitrile, under VUV photons irradiation of interstellar/pre-cometary ice analogs. From infrared spectroscopy data, acetonitrile is formed from VUV irradiation of ethylamine at $20 \mathrm{~K}$ through a two step dehydrogenation process. Once formed, acetonitrile can then react under VUV irradiation in solid phase with ammonia leading to the formation of amino acetonitrile. Acetonitrile synthesis from ethylamine can be suggested as a possible pathway for its formation on interstellar or cometary grains. We emphasize that this scenario is at present the only one demonstrated in the laboratory and astrophysically relevant environments. Other formation pathways, in particular those related to solid/gas phases reactivity, which are possible in evaporating comets, can be proposed as alternative pathways. Finally these studies clearly show the vast complexity of photo and thermochemistry that may happen on astrophysical ices in various environments. They also clearly demonstrate the necessity of laboratory simulations whose diagnostics provide other information than a simple comparison with astrophysical data. These results have then to be discussed within scenarios proposed for the general evolution of interstellar/cometary dirty ices.

Acknowledgements. This work has been founded by the French national programme Physique Chimie du Milieu Interstellaire (P.C.M.I) and the Centre National des Études Spatiales (C.N.E.S).

\section{References}

Atreya, S. K. 1992, Adv. Space Res., 12, 1

Basiuk, V. A., \& Bogillo, V. I. 2002a, Adv. Space Res., 30, 1439

Basiuk, V. A., \& Bogillo, V. I. 2002b, Adv. Space Res., 30, 1445

Belloche, A., Menten, K. M., Comito, C., et al. 2008, A\&A, 482, 179

Belloche, A., Garrod, R. T., Muller, H. S. P., et al. 2009, A\&A, 499, 215

Bernstein, D. I., Sandford, S. A., Allamandola, L. J., \& Chang, S. 1995, ApJ, 454, 327

Bernstein, M. P., Dworkin, J. P., Sandford, S. A., Cooper, G. W., \& Allamandola, L. J. 2002, Nature, 416, 401

Bernstein, M. P., Ashbourn, S. F. M., Sandford, S. A., \& Allamandola, L. J. 2004, ApJ, 601, 365
Bossa, J. B., Duvernay, F., Theulé, P., et al. 2009a, A\&A, 506, 601

Bossa, J. B., Theulé, P., Duvernay, F., \& Chiavassa, T. 2009b, ApJ, 707, 1524

Codella, C., Benedettini, M. T. B. M., Gueth, F., et al. 2009, A\&A, 507, L25

d'Hendecourt, L. B., \& Allamandola, L. J. 1986, A\&ASS, 64, 453

Dartois, E. 2005, Space Sci. Rev., 119, 293

Dartois, E., \& d'Hendecourt, L. 2001, A\&A, 365, 144

Durig, J. R., Zheng, C., Gounev, T. K., Herrebout, W. A., \& van der Veken, J. 2006, J. Phys. Chem. A, 110, 5674

Duvernay, F., Chatron-Michaud, P., Borget, F., Birney, D. M., \& Chiavassa, T. 2007, Phys. Chem. Chem. Phys., 9, 1099

Duvernay, F., Dufauret, V., Danger, G., et al. 2010, A\&A, 523, A79

Elsila, J. E., Dworkin, J. P., Bernstein, M. P., Martin, M. P., \& Sandford, S. A. 2007, ApJ, 660, 911

Elsila, J. E., Glavin, D. P., \& Dworkin, J. P. 2009, Meteor. Planetary Sci., 44, 1323

Ferraro, J. R., Sill, G., \& Fink, U. 1980, Appl. Spectrosc., 34, 525

Gerakines, P. A., Schutte, W. A., \& Ehrenfreund, P. 1996, A\&A, 312, 289

Gerakines, P. A., Moore, M. H., \& Hudson, R. L. 2004, Icarus, 170, 202

Glavin, D. P., Dworkin, J. P., \& Sandford, S. A. 2008, Meteor. Planetary Sci., 43, 399

Hagemann, H., \& Bill, H. 1984, J. Chem. Phys., 80, 111

Hamada, Y., Hashiguchi, K., Tsuboi, M., Koga, Y., \& Kondo, S. 1984, J. Mol. Spectrosc., 105, 93

Hashiguchi, K., Hamada, Y., Tsuboi, M., Koga, Y., \& Kondo, S. 1984, J. Mol. Spectrosc., 105, 81

Hollis, J. M., Lovas, F. J., Remijan, A. J., et al. 2006, ApJ, 643, L25

Holtom, P. D., Bennett, C. J., Osamura, Y., Mason, N. J., \& Kaiser, R. I. 2005, ApJ, 626, 940

Hudson, R. L., \& Moore, M. H. 2004, Icarus, 172, 466

Hudson, R. L., Moore, M. H., Dworkin, J. P., Martin, M., \& Pozun, Z. D. 2008, Astrobiology, 8, 771

Huebner, W. B., Snyder, L. E., \& Buhl, D. 1974, Icarus, 23, 580

Jacox, M. E., \& Milligan, D. E. 1963, J. Am. Chem. Soc., 85, 278

Koch, D. M., Toubin, C., Peslherbe, G. H., \& Hynes, J. T. 2008, J. Phys. Chem. C, 112, 2972

Lara, L. M., Lellouch, E., Lopez-Moreno, J. J., \& Rodrigo, R. 1996, J. Geophys. Res., 101, 23261

Moore, M. H., \& Hudson, R. L. 2003, Icarus, 161, 486

Olmi, L., Cesaroni, R., \& Walmsley, C. M. 1993, A\&A, 276, 489

Oro, J. 1961, Nature, 190, 389

Remijan, A. J., Sutton, E. C., Snyder, L. E., et al. 2004, ApJ, 606, 917

Remijan, A. J., Milam, S. N., Womack, M., et al. 2008, ApJ, 689, 613

Rimola, A., Sodupe, M., \& Ugliengo, P. 2010, Phys. Chem. Chem. Phys., 12, 5285

Snyder, L. E., \& Buhl, D. 1971, ApJ, 163, L47

Sogoshi, N., Wakabayashi, T., Momose, T., \& Shida, T. 1997, J. Phys. Chem. A, 101,522

Spall, B. C., \& Steacie, E. W. R. 1957, Proc. R. Soc. London, Ser. A, 239, 1

Stolkin, I., Ha, T.-K., \& Gunthard, H. H. 1977, Chem. Phys., 21, 327

Venkateswarlu, P. 1951, J. Chem. Phys., 19, 293

Whittet, D. C. B., Gibb, E. L., \& Nummelin, A. 2001, Origins Life Evol. Biosphere, 31, 157

Woon, D. E. 2002, ApJ, 571, L177

Xu, S. C., \& Wang, N. 2007, Acta Phys. Chim. Sin., 23, 212

Zeroka, D., Jensen, J. O., \& Samuels, A. C. 1999, J. Mol. Struct. TheoChem, 465,119 